\title{
The influence of X-ray resonant Raman scattering effects on the detection of copper(II) tetrasulphonated phthalocyanine (CuTSPc) thin-films deposited on gold electrodes
}

\author{
Karl Peeters $^{\text {a }}$, Karolien De Wael ${ }^{\mathrm{a}, 1}$, Annemie Adriaens ${ }^{\mathrm{a}, *}$, Gerald Falkenberg ${ }^{\mathrm{b}}$, Laszlo Vincze ${ }^{\mathrm{a}}$ \\ ${ }^{\text {a }}$ Ghent University, Department of Analytical Chemistry, Krijgslaan 281 S12, B-9000 Ghent, Belgium \\ ${ }^{\mathrm{b}}$ Hamburger Synchrotronstrahlungslabor at Deutsches Elektronen-Synchrotron, DESY, D-22607 Hamburg, Germany
}

Received 7 October 2007; accepted 9 December 2007

Available online 23 December 2007

\begin{abstract}
The influence of X-ray resonant Raman scattering on the detection capabilities of copper(II) tetrasulphonated phthalocyanine (CuTSPc) thinfilms on gold electrodes has been investigated by means of synchrotron radiation X-ray fluorescence. In particular the paper focuses on the influence of the excitation energy in the relative narrow energy region of 9.2 to $11.7 \mathrm{keV}$ on the detection limits and the peak-to-background ratios of copper.
\end{abstract}

(C) 2007 Elsevier B.V. All rights reserved.

Keywords: X-ray resonant Raman scattering (XRRS); Detection limit; Copper(II) tetrasulphonated phthalocyanine (CuTSPc); Gold electrode; Synchrotron radiation $\mathrm{X}$-ray fluorescence (XRF)

\section{Introduction}

X-ray resonant Raman scattering (XRRS) is a phenomenon which occurs in the X-ray regime involving inner-core excitations. It takes place when the energy of the incident $\mathrm{X}$-ray radiation is smaller than the binding energy of an inner shell of the target atom [1]. The phenomenon was first demonstrated on solid targets by Sparks in 1974 and its principle is shown in Fig. $1[1,2]$. The binding energy of an inner shell electron can be described by a Lorentzian distribution due to the core hole lifetime broadening centered at the energy $E_{\mathrm{K}}$, the binding energy of the $\mathrm{K}$ electrons, with a full width at half maximum (FWHM) of $\Gamma_{K}$ [1]. When the energy of the incident $\mathrm{X}$-ray radiation approaches the binding energy of an inner shell electron of the target atom, a finite probability exists in which $\mathrm{K}$ shell electrons are excited above the Fermi-level and a virtual core hole is created. The virtual core hole is subsequently filled by an electron of a higher shell. The latter is followed by the

\footnotetext{
* Corresponding author. Tel.: +329264 4826; fax: +3292644960.

E-mail address: annemie.adriaens@ugent.be (A. Adriaens).

${ }^{1}$ Postdoctoral Fellow of the Research Foundation-Flanders (Belgium).
}

emission of a photon yielding a final state which is characterized by a core hole in a higher shell, an electron in the continuum and the emitted ("Raman scattered") photon. When the energy of the exciting radiation is close to that of an absorption edge of the atom, the process shows resonant behavior and is called the XRRS process [3-6]. The practical importance of the XRRS phenomenon has been recognized in the determination of ultra-trace impurities on substrates, such as the detection of low $\mathrm{Z}$ elements (e.g. Al, oxides) in silicon wafers by TXRF [7-10], Pt in ancient gold [11] and $\mathrm{Ni}$ in copper substrates [12]. In these cases the energies of the fluorescence lines of the impurities are just below the absorption edge of the main substrate or matrix element. The exciting photon energy is selected just below the absorption edge of the main substrate element to avoid the excitation of the substrate fluorescence which will otherwise dominate the X-ray fluorescence spectrum. Under such conditions the XRRS process results in a strong additional background contribution which may severely overlap with the fluorescence lines of the analyte element of interest. This affects the detection limits, net elemental yields as well as the accuracy of the quantification of the impurities. The latter can be improved by determining the 


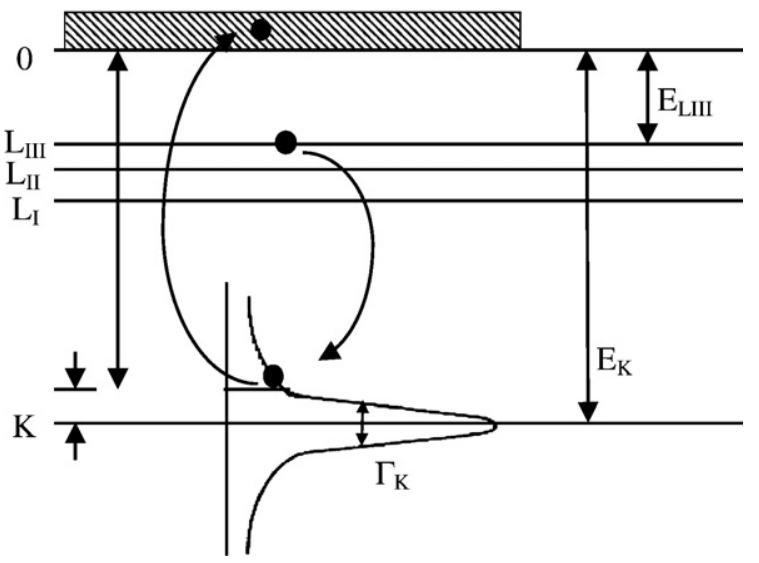

Fig. 1. A schematic energy diagram demonstrating the X-ray resonant Raman scattering process where $E_{\mathrm{K}}$ and $E_{\mathrm{LIII}}$ are the respective binding energies of the $\mathrm{K}$ and $L_{\mathrm{III}}$ shell electrons and $\Gamma_{\mathrm{K}}$ the full width at half maximum of the $\mathrm{K}$ shell electron due to core hole lifetime broadening. After [1].

cross-sections associated with the background distribution of the interfering XRRS process [12,13]. Another important effect which is difficult to discriminate from the resonant Raman contribution is, for example, the radiative Auger effect, which was pointed out by Van Espen et al. [14] and Kawai et al. [15].

In our study, the practical relevance of the XRRS phenomenon has been recognized during the quantitative investigation of the deposition properties of phthalocyanine thin-films on gold electrodes using synchrotron radiation X-ray fluorescence (SR-XRF) microspectroscopy. Transition metallo phthalocyanines are commonly used in high-tech applications, such as photosensitizers, in optical data storage and chemical gas sensors [16-20]. The application of phthalocyanines has also been expanded into various fields such as electrochemistry where they are frequently used as electrocatalysts [21-23]. Soluble sulphonated phthalocyanines have namely shown to be able to form layers on different types of supporting material, including electrodes [23-25]. The immobilization of these compounds on an electrode can lead to modified electrodes with improved kinetic properties. The major characteristics of the phthalocyanines are their high thermal and chemical stability, coupled with their extensive redox chemistry.

In this study, gold electrodes were modified with a copper(II) tetrasulphonated phthalocyanine tetrasodium salt (CuTSPc) using cyclic voltammetry. The method enables one to control and characterize the formed layer by following the potential and current during the process. The heterogeneity of the deposited thin-layer on the electrode was investigated using SR-XRF. The CuTSPc thin-film uniformity study implies the determination of low-amounts of $\mathrm{Cu}$ (ca. $0.1-0.5 \mathrm{pg}$, assuming a $20 \mu \mathrm{m}$ incident beam) [24] in the presence of a partially overlapping Au X-ray resonant Raman peak.

This paper discusses the influence of the XRRS contribution originating from the gold substrate on the copper detection in the excitation energy range of 9 to $12 \mathrm{keV}$, i.e. when the excitation energy approaches the $\mathrm{Au} \mathrm{L}_{3}$-edge. Results on the electrochemical behavior, the morphology and heterogeneity of the deposited thin-layer can be found in Peeters et al. [24].

\section{Experimental}

Gold electrodes were modified with a copper(II) tetrasulphonated phthalocyanine tetrasodium salt (CuTSPc, purchased from Sigma Aldrich, USA) by recording 60 successive cyclic voltammetric scans in a potential window from -1.2 to $0.6 \mathrm{~V}$ vs SCE ("potential cycling method") in a $0.1 \mathrm{~mol} \mathrm{~L}^{-1} \mathrm{NaOH} /$ $\mathrm{Na}_{2} \mathrm{HPO}_{4} \mathrm{pH} 12$ buffer solution containing $4 \mathrm{mmol} \mathrm{L} \mathrm{L}^{-1}$ CuTSPc. Details on the complete modification procedure can be found in literature [24].

The SR-XRF experiments were performed at Beam Line L of the DORIS-III storage ring, HASYLAB (Hamburg, Germany) [26]. This beamline is dedicated to micro XRF experiments using either white or monochromatic bending magnet excitation and mono- or polycapillary focusing, with routinely available beam sizes of $10-30 \mu \mathrm{m}$. The primary X-ray beam is generated by a $1.2 \mathrm{~T}$ bending magnet source which, given the machine energy of $4.465 \mathrm{GeV}$, provides a polychromatic spectral distribution with a critical energy of $16.6 \mathrm{keV}$. After initial low-energy filtering and collimation, the beam was monochromatized by a $\operatorname{Si}(111)$ fixed exit monochromator $\left(\Delta / E \approx 10^{-4}\right)$ and further collimated by a motorized cross-slit system. After monochromatization the beam was focused by a monolithic polycapillary half-lens (X-ray Optical Systems Inc., USA). This optic is designed specifically for parallel primary beams hence suitable for the focusing of low-divergence synchrotron beams. A microbeam size of about $20 \mu \mathrm{m}$ (FWHM) was obtained at the

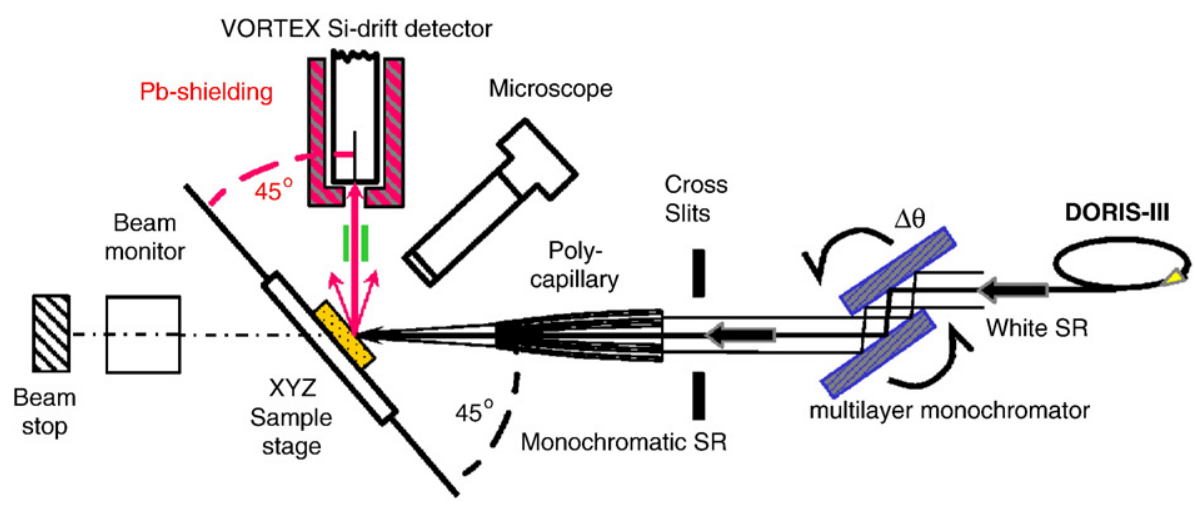

Fig. 2. Schematic representation of the micro SR-XRF instrument installed at HASYLAB Beam Line L (Hamburg, Germany). 


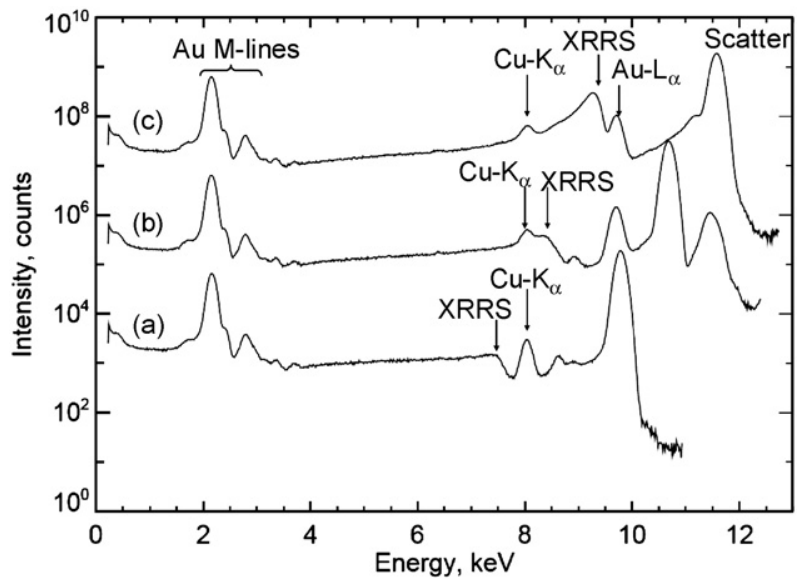

Fig. 3. Experimental SR-XRF spectra of the gold substrate/CuTSPc thin-film system, illustrating the evolution of the XRRS peak as the excitation energy varies from 9.8 (a) over 10.7 (b) to $11.6 \mathrm{keV}$ (c). The reduction of peak-tobackground ratios at copper $\mathrm{K}_{\alpha}$ towards higher energies can easily be observed. The top two SR-XRF spectra were multiplied by a factor $10^{2}$ and $10^{4}$ respectively for clarity. Note the presence of gold $\mathrm{L}_{\alpha}$ as a result of excitation by the $\mathrm{Si}(111)$ 3rd harmonic, which could only be partially suppressed by monochromator mistuning.

excitation energies. During this study the excitation energy was varied between $9.2 \mathrm{keV}$ and $11.7 \mathrm{keV}$, using a data collection time of $300 \mathrm{~s}$ for each individual SR-XRF spectrum. The nominal working distance of the polycapillary optic was $5 \mathrm{~mm}$. Fig. 2 shows the experimental arrangement used during the SRXRF study performed at beam line L.

The non-linear least square fitting software AXIL [27] was used to determine the net-peak intensities of the $\mathrm{Cu}-\mathrm{K}_{\alpha}$ and the background below this peak in the measured SR-XRF spectra. Pure thin $\mathrm{Cu}$ foils (thickness $1 \mu \mathrm{m}$ ) from Goodfellow Inc. were used as calibration standards. Quantification of the $\mathrm{Cu}$ surface concentration $C_{\mathrm{Cu}}$ at $9.3 \mathrm{keV}$ was performed using the calibration procedure described by Andreev et al. [22] and the detection limits (DL) for copper have been calculated according to the well-known relationship:

$\mathrm{DL}_{\mathrm{Cu}}=\frac{3 \sqrt{B_{\mathrm{Cu}-\mathrm{K} \alpha}}}{I_{\mathrm{Cu}-\mathrm{K} \alpha}} C_{\mathrm{Cu}}$

where $I_{\mathrm{Cu}-\mathrm{K} \alpha}$ and $B_{\mathrm{Cu}-\mathrm{K} \alpha}$ are the net-peak intensity and background of the $\mathrm{Cu}-\mathrm{K}_{\alpha}$ line, respectively, measured at the known surface concentration level of $C_{\mathrm{Cu}}$.

\section{Results and discussion}

In earlier scanning SR-XRF studies on CuTSPc thin-films on gold substrates, surface concentration detection limits in the range of 1-2 $\mathrm{ng} \mathrm{cm}^{-2}$ have been determined, assuming a live time of $300 \mathrm{~s}$ for data collection [24]. These detection limits are strongly influenced by the elemental yield of the analyte element (copper) and by the detected background. The latter mainly arises as a result of the various types of photon-matter interactions within the spectroscopically infinitely thick gold substrate. They include continuum radiation corresponding to photo-electron bremsstrahlung, the low-energy tail of the (multiple) Compton scattering peak just below the excitation energy and X-ray Raman scattering peaks/bands whose intensity is strongly influenced by the energy chosen for the monochromatic exciting beam.

Similar to analytical difficulties observed in the case of totalreflection XRF (TXRF) detection of aluminium impurities on silicon wafers [7], XRRS can also cause a considerable deterioration of detection limits in case of XRF analysis of transition-metal thin-films deposited on metallic substrates, such as gold electrodes. In our specific example, the efficient excitation of copper thin-films on gold substrates requires the selection of excitation energy above the $\mathrm{Cu}-\mathrm{K}$ absorption edge $(8.979 \mathrm{keV})$, while remaining below the $\mathrm{Au} \mathrm{L}_{3}$-edge $(11.918 \mathrm{keV})$ in order to achieve efficient excitation conditions for $\mathrm{Cu} \mathrm{K}$-lines and, at the same time, avoid detector overload by the orders of magnitude more intense Au L-lines.

Fig. 3 shows a series of experimental SR-XRF spectra obtained from CuTSPc thin-films electrochemically deposited on a gold electrode, corresponding to excitation energies in the energy range of 9.8-11.6 keV. While at lower energies $\left(E_{0} \leq 10 \mathrm{keV}\right)$, the XRRS contribution appears as a relatively small step-like discontinuity (below the copper peaks), it becomes an overwhelming and partially coinciding spectral feature when the excitation energy $\left(E_{0}\right)$ approaches the $\mathrm{Au} \mathrm{L}_{3}$-edge. Next to increasing detection limits (or reducing effective sensitivities), this XRRS interference can in special cases cause the detection of copper virtually impossible, due to the complete overlap of strong XRRS peaks with the $\mathrm{Cu}$ K-lines. Such condition of severe $\mathrm{Cu}-\mathrm{K}_{\alpha}$ and XRRS interference is shown in Fig. 4, in which case the excitation energy is chosen to be at the following energy:

$E_{0} \approx E\left(\mathrm{Cu}-\mathrm{K}_{\alpha}\right)+E\left(\mathrm{Au}-\mathrm{M}_{5}\right)$

where $E\left(\mathrm{Cu}-\mathrm{K}_{\alpha}\right)=8.04 \mathrm{keV}$ is the copper $\mathrm{K}_{\alpha}$ line energy, $E$ $\left(\mathrm{Au}-\mathrm{M}_{5}\right)=2.21 \mathrm{keV}$ the gold $\mathrm{M}_{5}$ absorption edge energy, respectively.

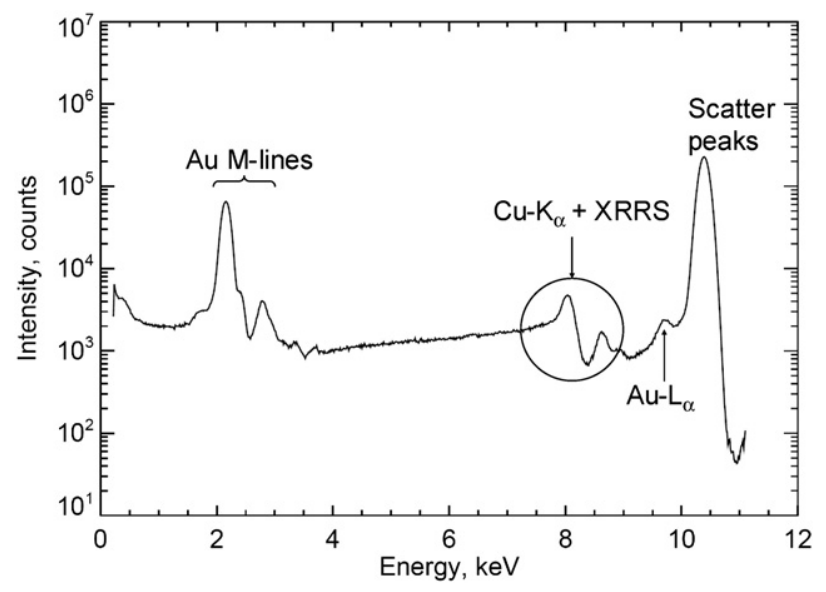

Fig. 4. Experimental SR-XRF spectrum showing the coinciding analyte line $\left(\mathrm{Cu}-\mathrm{K}_{\alpha}\right)$ and the resonant Raman band at an excitation energy of $10.40 \mathrm{keV}$. At this excitation energy, the separation of $\mathrm{Cu}-\mathrm{K}_{\alpha}$ and XRRS becomes virtually impossible. 

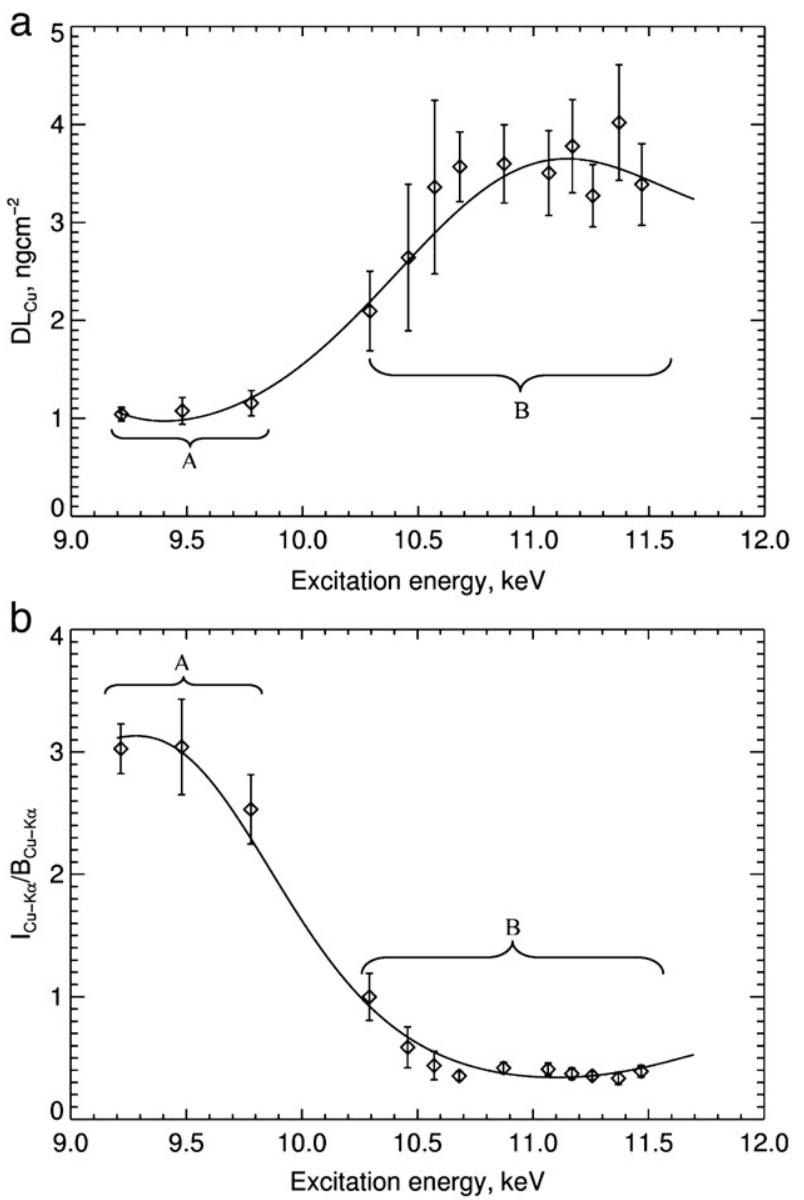

Fig. 5. Detection limits (a) and peak-to-background ratios (b) of $\mathrm{Cu}$ thin-films on an infinite thick gold substrate as a function of the excitation energy. The excitation energies with low detection limits and high peak-to-background ratios (A) and with high detection limits and low peak-to-background ratios (B) are grouped. In both cases the continuous line corresponds to a fitted (logarithmic) polynomial function which indicates the general trend as a function of incident beam energy.

At excitation energies above this region, the detection of copper becomes reliable again due to the shift of the XRRS peaks towards higher energies. However, detection limits progressively increase by the rapid increase of XRRS tailing and the reduction of copper excitation efficiency as $E_{0}$ becomes considerably higher than the $\mathrm{Cu} \mathrm{K}$-edge.

The variation of detection limits is shown in Fig. 5a as a function of excitation energy in the studied energy interval. These detection limits approximately increase by a factor 3.7 , from $1.0 \mathrm{ng} \mathrm{cm}^{-2}$ (A in Fig. 5a) to approximately $3.7 \mathrm{ng} \mathrm{cm}^{-2}$ (B in Fig. 5a), when the excitation energy is increased gradually in the energy range of $9.2-11.4 \mathrm{keV}$. This is mainly attributed to the considerable increase of overlapping spectral components as a result of XRRS, resulting in approximately a 10 -fold increase of the detected background level at $11.7 \mathrm{keV}$ compared to $9.2 \mathrm{keV}$. This effect is coupled with the decreasing excitation efficiency for copper towards higher $E_{0}$ values, resulting in an overall reduction of peak-to-background ratios as a function of growing excitation energy, as shown in Fig. 5b. In the energy region of 9.2-11.7 keV, the peak-to-background ratio changes from 3.10 (A in Fig. 5b) to 0.30 (B in Fig. 5b) which indicates a dramatic reduction of effective elemental yield for $\mathrm{Cu}$.

\section{Conclusions}

The work has focused on the effects of X-ray resonant Raman scattering (XRRS) in the context of its analytical influence on the outcome of scanning XRF measurements on CuTSPc thin-film detection on gold substrates using monochromatic synchrotron radiation. In the specific case of the detection of copper deposited on spectroscopically infinitely thick gold electrodes, the choice of excitation energy in the relatively narrow energy band of 9.2-11.7 keV has a great influence on the expected detection limits and peak-to-background ratios which can be observed during the XRF measurements. Detection limits range from 1.0 to $3.7 \mathrm{ng} \mathrm{cm}^{-2}$ as the excitation energy is varied between 9.2 and $11.4 \mathrm{keV}$, while peak-to-background ratios worsen by a factor of $\sim 10$ (from 3.10 to 0.30 ), indicating the strong interference effects of XRRS with respect to the detection of the CuTSPc layer. This study represents a new example of the often unexpected contribution of XRRS on the outcome SRXRF measurements using monochromatic X-rays for specific analyte/sample substrate combinations, such as transition-metal thin-film detection on gold electrodes.

\section{Acknowledgements}

This work was supported by the European CommunityResearch Infrastructure Action under the FP6 "Structuring the European Research Area" Programme (through the Integrated Infrastructure Initiative "integrating Activity on Synchrotron and Free Electron Laser Science") and Ghent University. The authors would like to acknowledge the help of K. Leyssens, T. Schoonjans and B. Schotte during the SR-XRF measurements.

\section{References}

[1] Y. Udagawa, H. Hayashi, K. Tohji, T. Mizushima, X-ray resonant Raman spectra of several copper compounds, J. Phys. Soc. Jap. 63 (1994) $1713-1720$.

[2] C. Sparks, Inelastic resonance emission of X-rays: anomalous scattering associated with anomalous dispersion, Phys. Rev. Lett. 33 (1974) 262-265.

[3] J.P. Briand, A. Simionovici, P. Chevallier, P. Indelicato, Infrared divergence of resonant Raman-Compton Scattering, Phys. Rev. Lett. 62 (1989) 2092-2095.

[4] A. Simionovici, J.P. Briand, P. Indelicato, P. Chevallier, Resonant RamanCompton scattering on zirconium, Phys. Rev. Lett. A 41 (1990) 3707-3715.

[5] P. Suortti, Scattering of X-rays near the K absorption-edge. 1. Fluorescence and resonant Raman scattering in transition-metals, Phys. Status Solidi, B-Basic Solid State Phys. 91 (1979) 657-666.

[6] S. Manninen, Resonant Raman scattering and fluorescence spectroscopy, Radiat. Phys. Chem. 50 (1997) 77-89.

[7] K. Baur, J. Kerner, S. Brennan, A. Singh, P. Pianetta, Aluminium impurities in silicon: investigation of X-ray Raman scattering in total reflection X-ray fluorescence spectroscopy, J. Appl. Phys. 88 (2000) 4642-4647.

[8] K. Baur, S. Brennan, B. Burrow, D. Werho, P. Pianetta, Laboratory and synchrotron radiation total-reflection X-ray fluorescence: new perspectives in total detection limits and data analysis, Spectrochim. Acta Part B 56 (2001) 2049-2056.

[9] C. Streli, G. Pepponi, P. Wobrauschek, B. Beckhoff, G. Ulm, S. Pahlke, L. Fabry, Th. Ehmann, B. Kangießer, W. Malzer, W. Jark, Analysis of low Z elements on $\mathrm{Si}$ wafer surfaces with undulator radiation induced total reflection X-ray fluorescence at the PTB beamline at BESSY II, Spectrochim. Acta Part B 58 (2003) 2113-2121. 
[10] J. Szlachetko, M. Berset, J.-Cl. Dousse, K. Fennane, M. Szlachetko, R. Barret, J. Hoszowska, A. Kubala-Kukus, M. Pajek, Resonant X-ray Raman scattering for Al, Si and their oxides, Nucl. Instrum. Methods Phys. Res., B 238 (2005) 353-356.

[11] M.F. Guerra, T. Calligaro, M. Radtke, I. Reiche, H. Reisemeier, Fingerprinting ancient gold by measuring Pt with spatially resolved high energy Sy-XRF, Nucl. Instrum. Methods Phys. Res., B 240 (2005) 505-511.

[12] Ch. Zarkadas, A.G. Karydas, M. Müller, B. Beckhoff, X-ray resonant Raman scattering on Ni employing polarized and unpolarized exciting radiation, Spectrochim. Acta Part B 61 (2006) 189-195.

[13] M. Müller, B. Beckhoff, G. Ulm, Absolute determination of cross section for resonant Raman scattering on silicon, Phys. Rev., A 74 (2006) 012702.

[14] P. Van Espen, H. Nullens, F. Adams, Radiative Auger effect in X-ray fluorescence analysis, Anal. Chem. 51 (1979) 1325-1328.

[15] J. Kawai, T. Nakajima, T. Inoue, H. Adachi, M. Yamaguchi, K. Maeda, S. Yabuki, Chemical effects on the intensity of Ti KH (radiative Auger satellite) x-ray fluorescence spectra, Analyst 119 (1994) 601-603.

[16] A.B.P. Lever, E.R. Milaeva, G. Speier, in: C.C. Leznoff, A.B.P. Lever (Eds.), Phthalocyanines properties and applications, vol. 3, Weinheim, 1993.

[17] A.B.P. Lever, The other periodic chart, Chemtech 17 (1987) 506-510.

[18] M. Kato, Y. Nishioka, K. Kaifu, K. Kawamura, S. Ohno, Near-infrared sensitive electro photographic photoconductors using chloroindium chlorophthalocyanine, Appl. Chem. Lett. 86 (1985) 196-197.

[19] J.E. Kuder, Organic active layer materials for optical recording, J. Imag. Sci. 32 (1988) 51-56.

[20] T.A. Temofonte, K.F. Schoch, Phthalocyanine semiconductor sensors for room temperature PPB level detection of toxic gases, J. Appl. Phys. 65 (1989) 1350-1355.
[21] K.J. Balkus, M. Eissa, R. Levado, Oxidation of alkanes catalyzed by zeolite-encapsulated perfluorinated ruthenium phthalocyanines, J. Am. Chem. Soc. 117 (1995) 10753-10754.

[22] A. Andreev, V. Ivanova, L. Prahov, I.D. Schopov, Catalytic activity of monomeric and polymeric cobalt(II) phthalocyanines in sulfide ion oxidation, J. Mol. Catal. 95 (1995) 197-201.

[23] K. De Wael, P. Westbroek, E. Temmerman, Electrocatalytic oxidation of dithionite at a cobalt(II) tetrasulfonated phthalocyanine and 5,10,15,20tetrakis-(4-sulphonatophenyl) porphyrin cobalt(II) modified gold electrode in alkaline solution, Electroanalysis 17 (2005) 263-268.

[24] K. Peeters, K. De Wael, A. Adriaens, G. Falkenberg, L. Vincze, Quantitative synchrotron micro-XRF study of CoTSPc and CuTSPc thin-films deposited on gold by electrodeposition, J. Anal. At. Spec. 22 (5) (2007) 493-501.

[25] K. De Wael, K. Peeters, D. Bogaert, H. Buschop, L. Vincze, A. Adriaens,

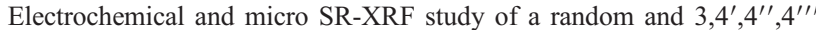
copper(II) tetrasulphonated phthalocyanine modified gold electrode, J. Electroanal. Chem. 603 (2007) 212-218.

[26] G. Falkenberg, O. Clauss, A. Swiderski, T. Tschentscher, Upgrade of the $\mathrm{X}$-ray fluorescence beam line at HASYLAB/DESY, X-Ray Spectrom. 30 (2001) 170-173.

[27] B. Vekemans, K. Janssens, L. Vincze, F. Adams, P. Vanespen, Analysis of X-ray-spectra by iterative least-square (AXIL) - new developments, X-Ray Spectrom. 23 (1994) 278-285. 